\title{
2009 Materials Research Society Spring Meeting Embraces Topics from Electronics to Energy See Expanded Meeting Coverage: www.mrs.org/S09MeetingReport
}

The 2009 Materials Research Society Spring Meeting, chaired by Paul R. Besser (CMOS Industry), Peter Fratzl (MaxPlanck-Institute of Colloids and Interfaces), Nicola Spaldin (University of California, Santa Barbara), and Terry M. Tritt (Clemson University), was held on April 13-17, 2009, in San Francisco, Calif. Through 41 symposia, symposium organizers from around the world expanded the standard Spring meeting focus on electronic materials and devices to also include coverage of developments in the areas of bio- and nanomaterials and materialsrelated energy issues. The Meeting included oral and poster presentations, award talks, an international equipment and resource exhibit, and special outreach and educational opportunities, including tutorials, professional development for women in materials science and engineering, and information on government funding and policymaking, including a tutorial on how to write grant proposals. This year, the Meeting Chairs also directed a spotlight on materials solutions to challenges in the developing world (see sidebar).

To carry through with the Society's imperative to use materials research to improve the quality of life, the meeting offered a cluster of symposia on energy and the environment. Symposium $R$ on renewable energy opened with a session on the global energy landscape, building on the recent MRS Bulletin issue, "Harnessing Materials for Energy" (April 2008). D. Steingart (City College of New York) started the session with a look at wireless sensor networks for energy and process monitoring. By measuring pressure, temperature, current, potential, humidity, and other parameters, sensors integrated into energy-intensive processes can help manage and save energy, he said. Additionally, energy from vibrations, waste heat, or sunlight can be harvested locally to power these devices, which operate well under $1 \mathrm{~mW}$. While harvesting energy is the long-term solution, Steingart said there is nothing wrong with using batteries or other power sources until the art of harvesting local energy is optimized. He gave an example of aluminum smelting plants, where monitoring heat and other param-

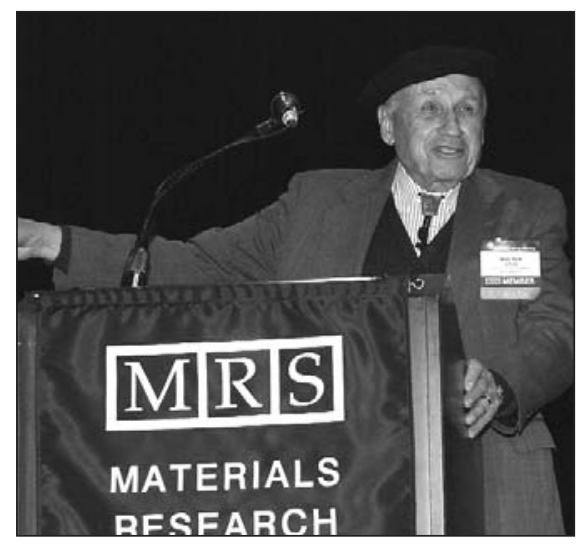

Nobel laureate Walter Kohn of the University of California, Santa Barbara gave the plenary address on the topic of materials challenges in solar energy.

eters can be used to adjust processes and increase plant efficiency beyond the typical $45-50 \%$ generally achieved today.

Determining a trajectory for energy technologies for the future requires an understanding of available resources from traditional and new sources. R. Sassoon from the Global Climate \& Energy Project at Stanford University presented the concept of "exergy," or the useful portion of energy that is available to do work, looking at all sources including the sun, wind, tides, fossil fuels, biofuels, nuclear materials, and geothermal resources. He explained that exergy is calculated from thermodynamic properties of a substance relative to the properties of a reference environment. He provided a Web site where a database and pictorial exergy and carbon flow charts can be found that gives a detailed view of available resources and carbon flows. Data in the maps include carriers, transformations, and accumulations: http:/ /gcep.stanford.edu/research/ exergy/data.html. "[The] data provide a framework for analyses of the impacts of various technological advances and policy initiatives that could enable or encourage increased exergy efficiency or new energy pathways," Sassoon said.

The energy topic attracting the most attention at the Spring Meeting was solar photovoltaics (PVs). While debates contin- ue to rage among researchers studying crystalline silicon, thin-film, and organic PVs, who are all eager to outpace the others in the grand energy scheme, all camps converge on the importance of addressing issues to scale up their technologies beyond the barely detectable fraction of the energy footprint where they now stand. J. Benner (Natl. Renewable Energy Lab.) and M. Mapes (Dept. of Energy) together gave a presentation on sustaining rapid growth in photovoltaics. Benner said that growth rates need to accelerate to move beyond being a successful business to becoming a significant energy source. They said that $40 \%$ annual growth rates were not only probable, but needed. While sunlight is the most abundant renewable energy source, even PVs, when scaled up to such levels, have been a target for critics. Thus beyond concerns of efficiency and cost, issues of sustainability of source materials, energy efficient processing of solar cells, and waste handling/recycling have become integral issues in PV development.

In the cluster of symposia addressing electronic and optical materials, which MRS Spring Meetings are noted for, topics included amorphous and polycrystalline thin-film silicon; molecular and organic electronics; complementary metal oxide semiconductor gate stack scaling; advanced interconnects for micro- and nanoelectronics; chemical-mechanical planarization; packaging, chip-package interactions, and solder materials challenges; high-throughput synthesis and measurement methods for rapid optimization and discovery of advanced materials; nonvolatile memories; engineered multiferroics; high-temperature photonic structures; and terahertz technology development.

Symposium D on advanced interconnects for micro- and nanoelectronics opened with a presentation by Q. Lin (IBM) on a photo-patternable low- $k$ dielectric that can reduce the number of steps for the fabrication of a patterned dielectric in the interconnect and reduce the fabrication cost. The photo-patternable low- $k$ material has the potential to replace the $\mathrm{SiCOH}$ dielectric in the fat-wire levels of the interconnect.

NeoSilicon is a novel functional material consisting of an ensemble of nanocrystal- 
line Si quantum dots with controlled dot size and inter-dot distance. In Symposium A, S. Oda (Tokyo Inst. of Technology, Japan) described the preparation of monodispersed Si nanocrystal quantum dots and their assembly, as well as the transport and optical properties of NeoSilicon. The bandgap of NeoSilicon can be controlled by dot size, due to the quantum size effect, while the transport properties can be controlled by the interdot distance due to the tunneling effect. Spherically shaped Si nanocrystals were formed by plasma decomposition of silane gas in a plasma cell, extracted from the plasma cell through a small orifice by a pressure difference and then deposited on a substrate at room temperature. These features are ideal for deposition of NeoSilicon onto large-area, flexible substrates. The surface of each Si nanocrystal is readily covered by its natural oxide or nitride, which serves as an ideal potential barrier and tunneling barrier with a very high-quality interface. The thickness of the

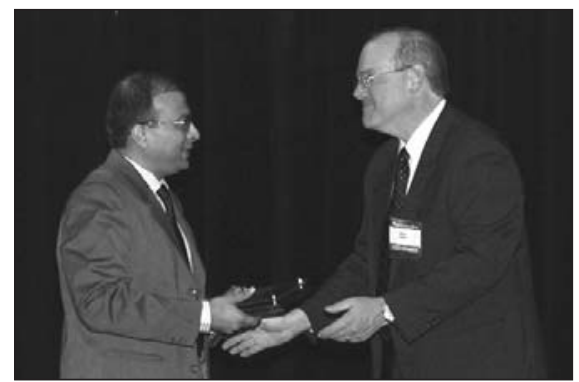

Anshu Bharadwaj (left), director of Center for Study of Science, Technology and Policy in Bangalore, India, accepts on behalf of V.S. Arunachalam (CSTEP) the MRS Board Special Recognition Award honoring his work on the special MRS Bulletin issue "Harnessing Materials for Energy" (April 2008). MRS President Shef Baker (right) presented the award.

surface layer determines the transport properties. The NeoSilicon materials show unique characteristics including Coulomb blockade, visible luminescence, and highefficiency electron emission. Oda also said that surface modification plays a key role in assembly and electron transport. The $\mathrm{Si}$ nanodots appear to be very promising for thin-film transistor and photovoltaics.

In Symposium I on engineered multiferroics, N.D. Mathur (Univ. of Cambridge, UK) described the development of inexpensive multilayered capacitors that have been labeled "the one-cent capacitor." Mathur's group recently showed that industrially manufactured multilayer capacitors based on $\mathrm{BaTiO}_{3}$ display strainmediated magnetoelectric effects because their electrodes now contain nickel, which is cheaper than the Ag-Pd alloy it replaces. Mathur described the magnetic and elastic response of these capacitors to an electric field, reporting ac magnetoelectric effects in multilayer capacitors. An interesting aspect of this work is the development of an undergraduate practical based on the magnetoelectric sensor, which can be used to explore ferroelectricity, ferromagnetism,
Cluster: Electronic and Optical Materials

Symposium A: Amorphous and Polycrystalline Thin-Film Silicon Science and Technology

Symposium B: Concepts in Molecular and Organic Electronics

Symposium C: CMOS Gate-Stack Scaling-Materials, Interfaces, and Reliability Implications

Symposium D: Materials, Processes, and Reliability for Advanced Interconnects for Micro- and Nano-Electronics

Symposium E: Science and Technology of Chemical Mechanical Planarization (CMP)

Symposium F: Packaging, Chip-Package Interactions, and Solder Materials Challenges

Symposium G: High-Throughput Synthesis and Measurement Methods for Rapid Optimization and Discovery of Advanced Materials

Symposium H: Materials and Physics for Nonvolatile Memories

Symposium I: Engineered Multiferroics-Magnetoelectric Interactions, Sensors, and Devices

Symposium J: High-Temperature Photonic Structures

Symposium K: Materials Research for Terahertz Technology

Development

Cluster: Energy and the Environment

Symposium L: Nuclear Radiation Detection Materials

Symposium M: Thin-Film Compound Semiconductor Photovoltaics

Symposium N: Materials and Devices for Thermal-to-Electric Energy Conversion

Symposium O: Compound Semiconductors for Energy Applications and Environmental Sustainability

Symposium P: Three-Dimensional Architectures for Energy Generation and Storage

Symposium Q: Materials Science of Water Purification

Symposium R: Materials for Renewable Energy at the Society and Technology Nexus

Symposium S: Materials in Photocatalysis and Photoelectrochemistry for Environmental Applications and $\mathrm{H}_{2}$ Generation

Cluster: Nanomaterials and Devices

Symposium T: Nanoscale Heat Transport-From Fundamentals to Devices

Symposium U: Electrofluidic Materials and ApplicationsMicro/Biofluidics, Electrowetting, and Electrospinning
Symposium V: Functional Metal-Oxide Nanostructures

Symposium W: Novel Functional Properties at Oxide-Oxide Interfaces Symposium Y: Nanocrystalline Materials as Precursors for Complex Multifunctional Structures through Chemical Transformations and Self Assembly

Symposium Z: Computational Nanoscience-How to Exploit Synergy between Predictive Simulations and Experiment

Symposium AA: Semiconductor Nanowires-Growth, Size-Dependent Properties, and Applications

Symposium BB: Material Systems and Processes for ThreeDimensional Micro- and Nanoscale Fabrication and Lithography

Symposium CC: Nanoscale Functionalization and New Discoveries in Modern Superconductivity

Symposium DD: Ion Beams and Nano-Engineering

Symposium EE: Materials for Nanophotonics_-Plasmonics, Metamaterials, and Light Localization

Symposium FF: Novel Materials and Devices for Spintronics Symposium GG: Electron Crystallography for Materials Research

Symposium HH: Quantitative Characterization of Nanostructured Materials

Symposium II: Probing Mechanics at Nanoscale Dimensions Symposium JJ: Nanoscale Electromechanics and Piezoresponse Force Microscopy of Inorganic, Macromolecular, and Biological Systems

Cluster: Soft Matter, Biological and Bio-Inspired Materials Symposium KK: Structure-Property Relationships in Biomineralized and Biomimetic Composites

Symposium LL: Architectured Multifunctional Materials

Symposium MM:Synthesis of Bio-inspired Hierarchical Soft and Hybrid Materials

Symposium NN: Active Polymers

Symposium OO: Materials and Strategies for Lab-on-a-Chip-Biological Analysis, Cell-Material Interfaces, and Fluidic Assembly of Nanostructures

Symposium PP: Materials and Devices for Flexible and Stretchable Electronics

Cluster: General Interest

Symposium X: Frontiers of Materials Research 
magnetostriction, magnetoelectric coupling, piezoelectricity, and pyroelectricity by using a mounted multilayer capacitor, a multimeter, and a magnet.

The Spring Meeting's traditional focus on electronic materials and devices continued in the cluster of symposia on nanomaterials and devices. Recently, diamondbased materials have emerged as a unique platform for quantum science and engineering. Spins of single nitrogen-vacancy (NV) color centers in diamond can be imaged, initialized, and read-out optically at room temperature, and measurements show remarkably long room-temperature electron spin coherence times well into the microsecond regime. In his talk in Symposium FF, D.D. Awschalom (Univ. of California, Santa Barbara) described the coherent control and coupling of single spins in diamond. He said that while the value of a (gem) diamond was based on the four Cs (color, clarity, carat, and cut), the value of diamond for spintronics was based on coherence, control, communication, and coupling.

Using magneto-optical imaging and electron spin resonance (ESR) measurements, single NV center spins that are coupled to electron spins of nearby nitrogen $(\mathrm{N})$ defects were investigated. Some of the NV centers are strongly coupled to only one single $\mathrm{N}$ spin, allowing controlled polarization and readout of this single "dark" N spin with remarkably long lifetimes. Also, room-temperature coherent control of the NV center spin using optical detection of pulsed ESR and spin echo techniques was shown. Diamond was also used to study the coherent dynamics of a single central spin coupled to an adjustable bath of spins. These experiments revealed that both the internal interactions of the bath and the coupling between the central spin and the bath can be tuned in situ with an applied magnetic field, allowing access to regimes with surprisingly different behavior. Combining these elements with precise implanting techniques paves the way toward future devices based on quantum coherent control of multiple coupled spins in diamond, Awschalom said.

J.T. Foukas (Univ. of Maryland) introduced an approach to photolithography that enables complex three-dimensional (3D) nanostructures having a feature size as small as one-twentieth of the excitation wavelength to be patterned in a single photo-exposure step. The method, named resolution augmentation through photoinduced deactivation (RAPID), demonstrates a means for overcoming the diffraction limit in optical lithography. Foukas's group showed that multiphoton-initiated polymerization activated at $800 \mathrm{~nm}$ using a focused laser beam can be suppressed by overlapping a second deactivation beam in the pre-polymer. When the deactivation beam is spatially shaped, the photo-polymerization volume can be shrunk much smaller than the diffraction limit. Remarkably, the deactivation beam can be a long-pulse or continuous wave beam also tuned to $800 \mathrm{~nm}$, so RAPID can be implemented with a single laser. Because multiphoton excitation can be used to direct-write 3D structures, the
Fourkas group was able to create 3D nanostructures with axial features as small as $40 \mathrm{~nm}$.

Coupling between electrical signals and mechanical motion in biological systems is essential to life and many biopolymers are in fact piezoelectric. Little is known, however, about the effect of electrical stimuli on the chemistry and functionality of biological systems, particularly at the nanoscale. B.J. Rodriguez (Univ. College Dublin, Ireland) probed electromechanical coupling in biological systems, as dis-

\section{Graduate Students Receive Gold and Silver Awards}

Graduate Student Awards were announced during an evening ceremony on April 15 at the 2009 Materials Research Society Spring Meeting in San Francisco.

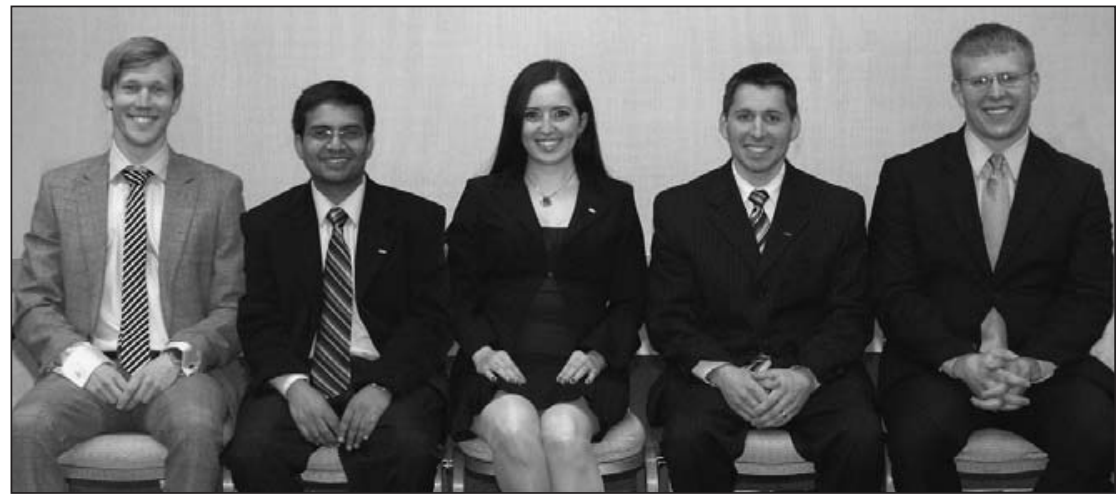

Gold Graduate Student Awards were awarded to (left to right): Magnus P. Jonsson (Chalmers University of Technology), Bishnu P. Khanal (Rice University), Ayse Asatekin (Massachusetts Institute of Technology), Erik C. Garnett (University of California, Berkeley), and Matthew J. Bierman (University of Wisconsin, Madison).

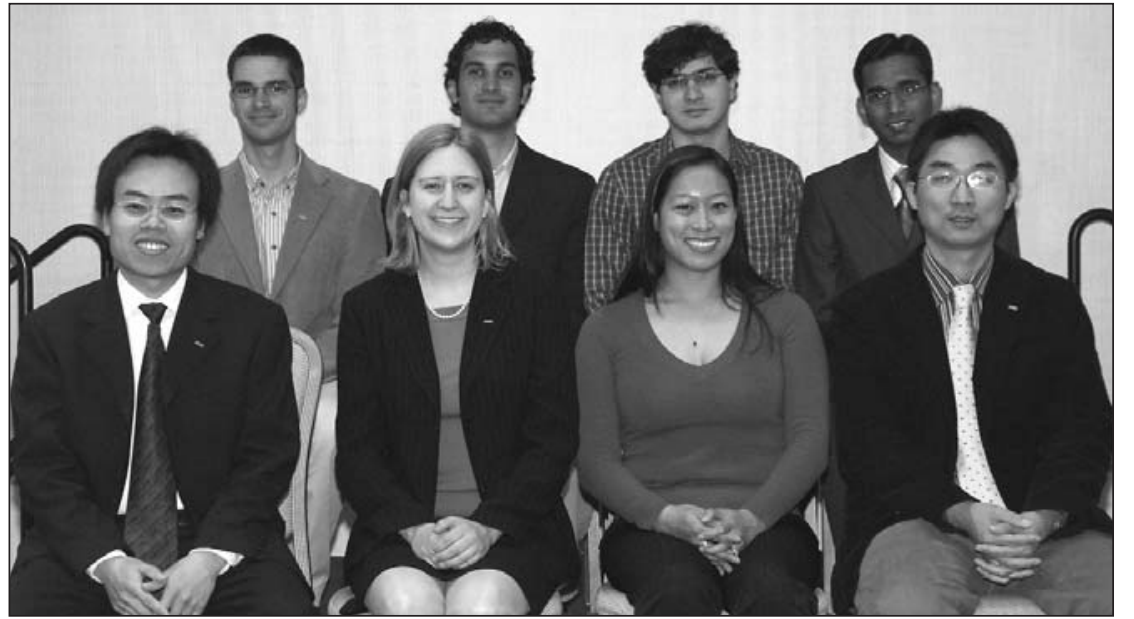

Silver Graduate Student Awards were awarded to (front row, left to right): Xiaolong Luo (University of Maryland), Kelly A. Burke (Case Western Reserve University), Candace K. Chan (Stanford University), and Jian Shen (University of California, San Diego); and (back row, left to right): Darren J. Lipomi (Harvard University), Sinan Keten (Massachusetts Institute of Technology), Bahman Hekmatshoar (Princeton University), and Purushottam Kumar (University of Florida). 
cussed in a talk in Symposium JJ. Studying electromechanical coupling in biological systems requires the ability to apply bias in physiological environments and to measure small displacements at the cellular and molecular level of soft materials. Piezoresponse force microscopy (PFM) is widely used to probe electromechanical phenomena in ferroelectric materials, and in recent years, PFM has been employed to study piezoelectric biomaterials. Rodriguez presented examples of electromechanical imaging of cellular and bio- molecular systems, including human teeth (dentin versus enamel) and collagen as well as other tissues. Nanoscale piezoelectricity is ubiquitous in biosystems due to the combination of polar bonds and optical activity in biopolymers. Rodriguez also discussed PFM of cellular systems including red blood cells and yeast cells. He mentioned the use of liquid PFM of cells in solution. Work is currently ongoing to address separation of the electromechanical response from electrostatic, elastic, and topographic contributions, and

\section{Poster Prizes Awarded at 2009 MRS Spring Meeting}

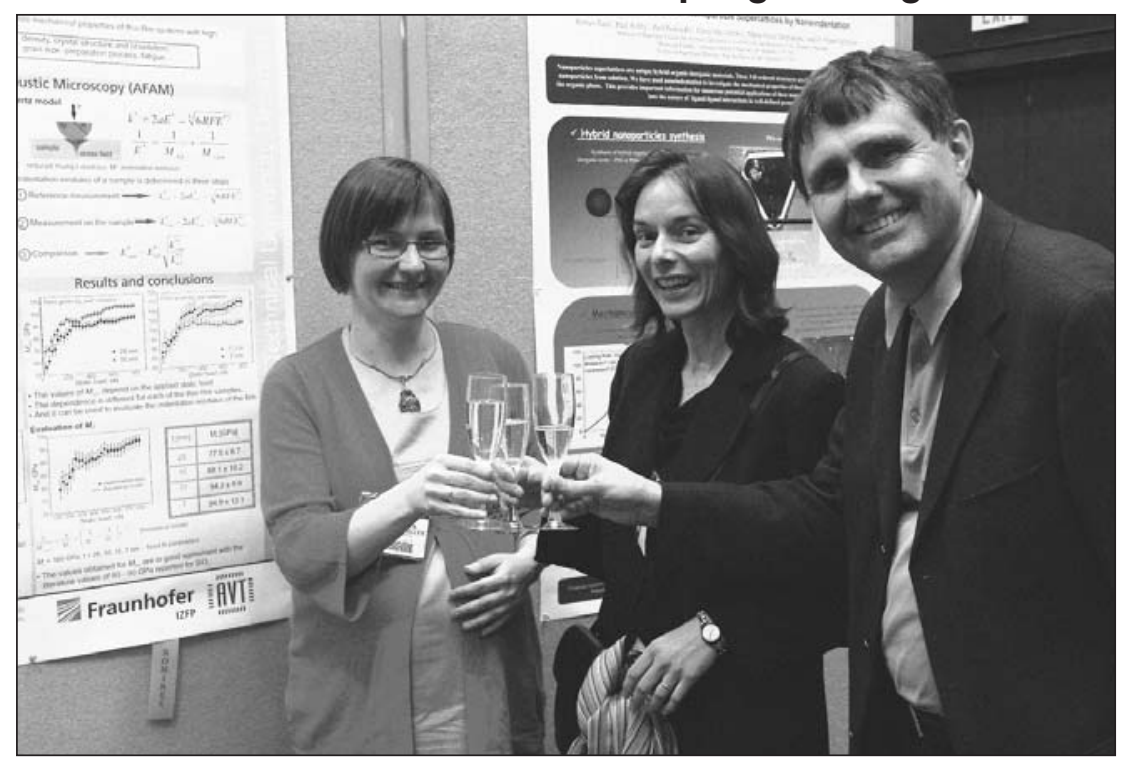

The 2009 Spring Meeting Chairs awarded prizes for the following best poster presentations: (A18.2) Structural Properties of a-Si:H Films with Improved Stability against Light Induced Degradation, G. van Elzakker and M. Zeman (Delft University of Technology) and P. Sutta (University of West Bohemia); (N3.3) Microstructure of Multiphase Thermoelectric Ag-Sb-Te Alloys, J.D. Sugar, P.A. Sharma, and D.L. Medlin (Sandia National Laboratories, Livermore); (M5.18) Enhanced Conversion Efficiency of GaAs Photovoltaics Utilizing AntiReflective Indium-Tin-Oxide Nano-Columns, C.-H. Chang, P. Yu, C.-H. Chiu, and H.-C. Kuo (National Chiao-Tung University) and M.-H. Hsu (National TsingHua University); (V9.35) Self Assembling Hybrid Materials for Solar Cells, M.C. Lechmann (Max Planck Institute) and J.S. Gutmann (MPI and University Mainz) ; (EE9.15) Modal Decomposition of Surface Plasmon Whispering Gallery Resonators, E.J.R. Vesseur and A. Polman (AMOLF) and J. Garcia de Abajo (CSIC, Madrid); (AA3.9) In Situ Catalytic Growth of Gallium Nitride Nanowires, R.E. Diaz, R. Sharma, S. Mahajan, and K. Jarvis (Arizona State University); (II9.4) Mechanical Properties of Nano-Thin Films by Use of Atomic Force Acoustic Microscopy, B. Koehler and N. Meyendorf (Fraunhofer Institute for NonDestructive Testing, Dresden-IZFP-D), M. Kopycinska-Mueller and A. Striegler (IZFP-D and Technical University Dresden), K.-J. Wolter (Technical University Dresden), and A. Huerrich (Fraunhofer Institute for Photonic Microsystems, Dresden); (KK5.18) The Effect of Silk Fibroin Hydrogels, Peptides, and $\beta$-chitin on Calcium Carbonate Crystallization: A Synthetic Model for Nacre Formation, E.C. Keene and L.A. Estroff (Cornell University) and J.S. Evans (New York University); and (OO3.15) Three-Dimensional Nano-Bio-Diagnostic System using Protein Nanoparticle, E.J. Lee, J.-S. Park, K.-Y. Ahn, M.K. Cho, H.-S. Seo, J.-A. Song, J.-H. Lee, J.-U. Shin, S.-E. Kim, Y.K. Kim, and J. Lee (Korea University). to relate the electromechanical response to biofunctionality.

Delving further into the realm of soft materials, the cluster on soft matter, biological, and bio-inspired materials included symposia on biomineralized and biomimetic composites, and materials and strategies for lab-on-a-chip. In Symposium KK that included presentations on biomimetic composites, H. Birkedal from Aarhus University, Denmark, described the structural characterization of the sucker rings from the Humboldt squid, Dosidicus gigas. The squid, also called the red devil, is a large, aggressive, and predatory species commonly encountered throughout the Eastern Pacific. It has eight arms and two tentacles with suckers. Within the suckers are sucker rings consisting of rigid-toothed, ring-like structures that provide additional gripping power during prey capture and handling. Birkedal showed that the sucker rings from this species exhibit a unique set of characteristics not reported previously for any other biological structural material. They consist of a proteinaceous nanoscale network of densely packed parallel tubular elements that are presumably stabilized almost entirely by hydrogen bonding and hydrophobic interactions. The network of channels exhibits a distinctive mesostructure and organizational gradient, reducing its diameter and abundance from the tooth core to the periphery. Mechanical properties were investigated using nanoindentation and modeling studies. It was found that the nanostructure modulates the mechanical properties. Interestingly, the structure is completely protein-based.

In his talk on color-changing shapememory polymers (SMP) in Symposium NN, P.T. Mather (Syracuse Univ.) described the design, synthesis, processing, and characterization of new SMPs with built-in temperature-sensing capabilities. He prepared the new materials by incorporating a fluorescent, chromogenic dye into a family of crosslinked poly(cycloocteneco-cyclooctadiene) $\mathrm{P}(\mathrm{CO}-\mathrm{co}-\mathrm{COD})$ matrices by imbibing from a dye solution. The new SMP copolymers were designed to feature tailored melting temperature and were prepared by ring-opening metathesis polymerization through ruthenium catalysis, followed by thermal crosslinking with dicumyl peroxide. The dye concentration was chosen to allow for self-assembly of the dye upon drying, resulting in the formation of excimers. Heating the phaseseparated blends to temperatures above the SMP melting point caused dissolution of the dye molecules and associated change optical absorption and fluorescence 
color. These changes are reversible, and the transition is determined by copolymer composition. Mather also described endlinked shape memory networks. Mather concluded that covalently crosslinked crystalline SMPs offer excellent strain fixing and recovery. Both one-way and two-way shape memory effects are possible with crystalline networks. Color change in SMPs is possible by incorporation of an excimer-forming dye, and this effect seems broadly applicable to crystalline SMPs. In future work, Mather plans to exploit this color-changing effect for sensing applications as well as in SMP science.

In the area of lab-on-a-chip, covered in Symposium OO, nanopores allow for the confinement and study of single macromolecules (such as DNA) in the nanometer scale with a temporal resolution of some microseconds. B. Schiedt (Natl. Ctr. for Scientific Research-Lab. for Photonics and Nanostructures, Marcoussis, France) described the use of a focused ion beam (FIB) system to form a hole directly at a specified location on a film for a single nanopore device. The pore size for such a device needs to be comparable to the size of the molecule to be analyzed. It should also allow for the ability to add further functionality and should be reproducible in quantities. Schiedt's group thus used a nanoFIB and an automated patterning platform for this purpose. After a single nanopore was formed, four lines were inscribed around it (forming a cross) using a low dose to be able to locate the pore in a transmission electron microscope or even an optical microscope. The researchers were able to form an $8 \mathrm{~nm}$ pore in $50-\mathrm{nm}$ thick $\mathrm{SiN}$ and a $3 \mathrm{~nm}$ pore in a $20-\mathrm{nm}$ thick $\mathrm{SiC}$. Schiedt reported the first translocation results for a DNA molecule and a fibronectin molecule, thus demonstrating the successful use of such single nanopore devices for single-molecule biosensing.

\section{Outstanding Young Investigator Teri W. Odom Discusses Quasi-3D Plasmonic Lattices}

Teri W. Odom of Northwestern University is the recipient of the 2009 MRS Outstanding Young Investigator award. She presented her award lecture on quasi3D plasmonic lattices as part of Symposium EE. Surface plasmon polaritons (SPPs) are responsible for optical phenomena including negative refraction, enhanced optical transmission, and nanoscale focusing of light. Although many materials support SPPs, the choice of metal for most applications has been based on traditional plasmonic materials

\section{Materials Offer Promising Solutions to Challenges in the Developing World}

True to the MRS mission of researching materials to improve the quality of life, the 2009 MRS Spring Meeting illuminated the special needs and opportunities for materials-related activities in underdeveloped areas of the globe. Presentations spanned from an exhibit of devices to serve immediate life-saving measures to lectures illustrating far-reaching solutions informed by materials.

According to the company Vestergaard Frandsen, in Denmark, 6,000 people die every day from waterborne diseases. To combat this problem, Vestergaard Frandsen has invented the LifeStraw ${ }^{\circledR}$ Personal portable water purifier, designed to provide clean and safe drinking water to people outside their homes (see photo).

Researchers in Lehigh University's Environmental Engineering Program have synthesized two materials to address toxic water: a hybrid nanosorbent, ArsenX, which removes arsenic from contaminated groundwater, and a Hybrid Inorganic Material capable of sensing the presence of toxic metals (e.g., $\mathrm{Pb}, \mathrm{Cu}$, and $\mathrm{Zn}$ ) in drinking water simply through $\mathrm{pH}$ changes.

Researchers at the University of Central Florida Environmental Chemistry Laboratory and the National Aeronautics and Space Administration at Kennedy Space Center have collaborated for almost 15 years, developing many innovative technologies for a clean environment, including purification of water. One of their technologies is the Emulsified Zero Valent Iron, which is a biodegradable material that can be either pumped into the contaminated aquifer or used to treat water above ground with no maintenance or staff required after application.

In the technical session Symposium Q, on Materials Science of Water Purification, researchers elaborated on current research underway. For example, Maria M. Fidalgo de Cortalezzi (Buenos Aires Institute of Technology, Argentina) described the use of nanostructured iron oxide in water treatment. Iron oxides are low cost and well-studied materials that have been applied extensively in traditional water treatment processes such as coagulation. Now the availability of these materials in the nanoscale has made them interesting for a wider array of applications, Fidalgo de Cortalezzi said. In recent years, she has investigated iron oxide nanoparticles as base materials for the fabrication of new devices and processes for the treatment of drinking water. Iron oxide nanoparticles were used as the precursor for high-specific-surface-area ceramics. Their adsorption properties for arsenic and other heavy metals were studied in a variety of conditions and water matrices, and the material was characterized extensively. Based on this, her research team has developed a lowcost arsenic adsorbent device tailored for application in rural and remote areas with minimum requirements. A membrane-adsorbent reactor was also designed to more efficiently use the material for applications of high water demand such as municipalities.

Water samples from naturally arsenic-contaminated groundwater in the Buenos Aires province in Argentina were used to perform field tests of the systems. The study showed that the good performance observed in artificial arsenic solutions was maintained for the natural samples and allowed for a preliminary cost assessment of the technology. The iron oxide ceramics were also investigated as ultrafiltration membranes. Retention and fouling potential of different organic macromolecules

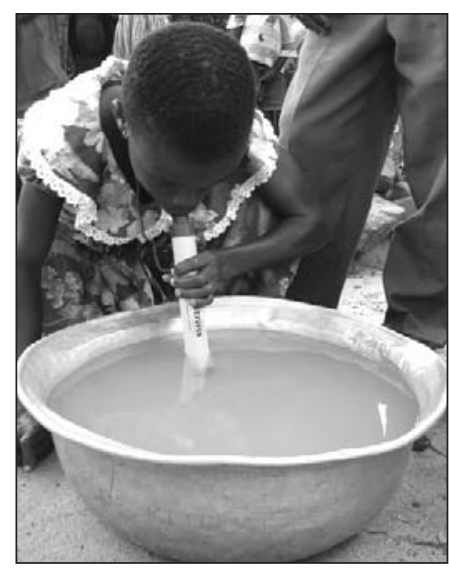

The LifeStraw ${ }^{\circledR}$ Personal portable water purifier, designed to provide clean and safe drinking water to people outside their homes, was displayed by Vestergaard Frandsen in a special exhibit on materials for the developing world during the MRS Spring Meeting. were measured. Work in this area focused on the evaluation of new cleaning methods for the ceramics through degradation of the adsorbed foulants by Fenton type reactions. Cleaning of the fouled membrane with a Fenton reagent recovered $100 \%$ of the initial membrane permeability, with no detectable membrane material loss, Fidalgo de Cortalezzi reported.

Engineers Without Borders, based at the University of California, Santa Barbara, and a special undergraduate study-abroad program in the Department of Materials Science and Engineering at Iowa State University were also represented. The key to both programs is for students to apply the principles of technology that is appropriate to the environmental, cultural, and economic situation for (Continued on page 612) 
such as $\mathrm{Ag}$ and Au because there have been no side-by-side comparisons of different materials on well-defined, nanostructured surfaces.

Odom's group has developed a multiscale patterning approach-soft interference lithography combined with PEELthat can be used to create a wide range of hierarchically structured, plasmonic materials. PEEL is a simple procedure that combines Phase-shifting photolithography, Etching, Electron-beam deposition, and Lift-off of the metal film. Typical patterned areas exceed $1 \mathrm{in}^{2}$ with ca. $10^{10}$ nanostructures generated simultaneously.

Using these nanofabrication techniques, Odom and her co-workers have discovered that near-infinite arrays of $100-\mathrm{nm}$ holes in $\mathrm{Au}$ and $\mathrm{Pd}$ films exhibit ultrasharp spectral features that can be tuned by changing the material, dielectric envi- ronment, and excitation conditions. Significantly, the origin of these complex transmission spectra can be described by a new optical coupling mechanism that is valid for strong and weakly plasmonic materials. Dispersion diagrams of these artificially structured materials were analogous to electronic materials.

The nanofabrication platform described by Odom not only enables rapid screening of a wide range of metals under different excitation conditions, but can also identify unexpected materials and achieve high sensitivities for biosensing. Nanopyramidal gratings - with quasi-3D unit cellswere used to generate SPP dispersion diagrams for $\mathrm{Al}, \mathrm{Ag}, \mathrm{Au}, \mathrm{Cu}$, and $\mathrm{Pd}$. Surprisingly, the SPP coupling efficiencies of $\mathrm{Cu}$ and $\mathrm{Al}$ exceeded widely used plasmonic materials under certain excitation conditions. Furthermore, grazing angle excitation can produce the highest refractive index sensitivities at optical frequencies because of extremely narrow SPP resonances (full-width-at-half-maximum $<6 \mathrm{~nm})$. The screening process also revealed that $\mathrm{Ag}$, with the highest sensitivity, is not necessarily the ideal material for detecting protein-ligand binding. Odom ended her presentation by describing her group's most recent results on using nanopyramidal gratings to determine the kinetics of protein-ligand interactions and comparing the results to those obtained by conventional techniques.

\section{Biomineralization Inspires New Nanofabrication Strategies}

Joanna Aizenberg of Harvard University received the 2009 Kavli Distinguished Lectureship in Nanoscience and presented her lecture at the MRS Spring Meeting.

\section{(Continued from page 611)}

which it is intended. For example, in a village in Mali-in western sub-Saharan Africa-students from Iowa State University saw an opportunity to use materials to improve on the current cooking system used there. In a presentation in Symposium X, Richard LeSar of Iowa State University said Malians typically cook over a three-stone open fire, using biomass for fuel, which is inefficient, smoky, and dangerous in their homes. The students worked with the villagers to assess their needs, limitations, and available resources. Together they designed stoves made out of straw and clay. Straw and mud bricks provided the structure and insulating properties, and clay from termite mounds served as the facing

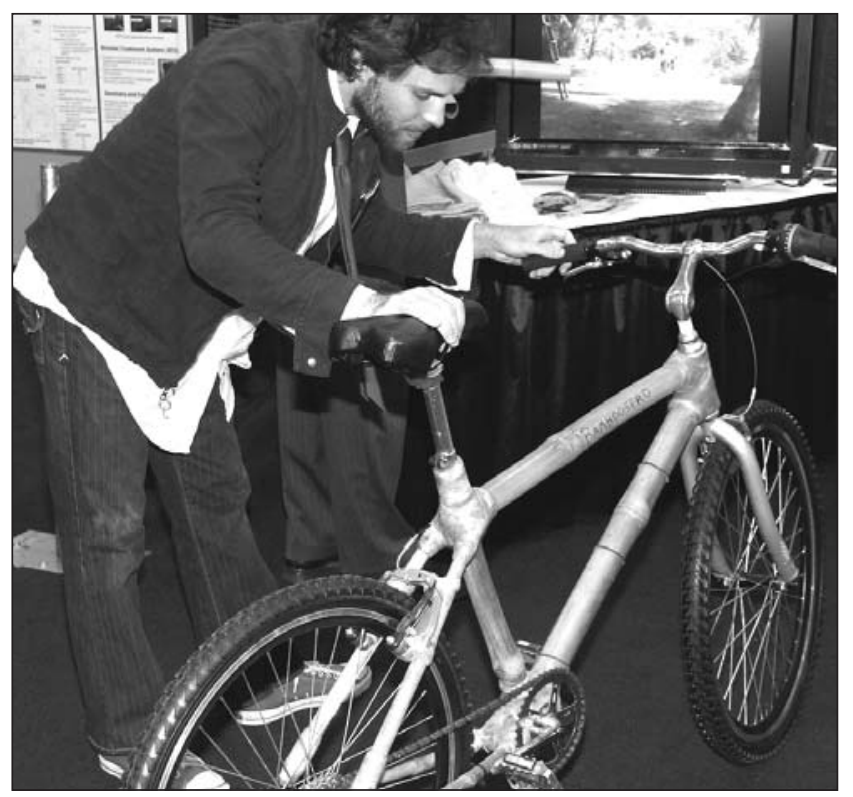

An MRS Spring Meeting attendee examines a bamboo bicycle made in Ghana by Bamboosero. The bicycle uses locally grown bamboo joined with a sisal/epoxy composite. The goal is to create self-sustaining businesses that help the developing world break the cycle of dependence on Western aid. material, which has a fine structure controlled by what termites can carry to the mound. They optimized parameters such as the spacing between stove and pot, and holes for the fuel, creating a more efficient stove, reducing fuel needs and emissions. While the Malians did not want the stoves at first, soon there was a waiting list to get them. LeSar said that a focus of the class is to avoid "drive-by" engineering, in which technologies are introduced into a developing country without a plan to sustain them. Rather, the class focuses on continued involvement for each project. To that end, technologies are developed in classes at the university and implemented in Africa, after which an assessment is made, which may lead to further development. LeSar said that over one-sixth of the 6.7 billion people in the world today live in extreme poverty, with incomes of less than a dollar a day. By 2050, the world will contain about nine billion people. LeSar uses his program to ask how materials engineering can help lead to a world with equitable and profitable economies by 2050 .

In the plenary address at the MRS Spring Meeting, Nobel laureate Walter Kohn of the University of California, Santa Barbara also addressed materials for the developing world. He said that he is very pleased with this theme at the conference, though he also wanted it to be noted that everyone is in "the same place" regardless of the distinction between "developed" and "developing." Kohn addressed the topic of materials challenges in solar energy. He screened his film, The Power of the Sun, which he produced in 2005. The film, narrated by British actor John Cleese, presented the history of society's understanding of light itself, from the initial wave hypothesis to the current wave-particle dual nature of light. The film traced the history of the development of the solar cell beginning at Bell Laboratories in the United States. The latter half of the film focused on the present scenario and the ever expanding use of solar energy through the world. Through the film, Kohn reiterated how solar energy is clean, sustainable, and environmentally friendly. In addition to the widespread use of silicon technology, other possibilities were also discussed, including organics. While challenges in large-scale adoption of the technology are immense, the film showed the success of solar energy in Japan and Germany as examples to emulate in other countries. One of the significant advantages of solar energy is the possibility of providing electricity in developing countries and, particularly, remote locations within such countries that are entirely off the power grid. It is possible to bring inexpensive and easily generated power to such places, allowing for improvements in the quality of life, Kohn said. 
Echinoderms - which are a phylum of marine animals, including the sea starand biomineralization have a lot to teach us in nanofabrication, she said. The calcitic skeleton in Echinoderms is made up of individual single crystalline elements with pores with controlled sizes. Aizenberg said this was used as the inspiration to grow oriented calcite crystals on self-assembled monolayers of organic molecules using stereochemical recognition. The critical issue to be noted here is that this is not epitaxy. Aizenberg described how ordered arrays of oriented uniform crystals can be formed. In Echinoderms, crystallization of complex structures often occurs through transformation from a transient amorphous phase stabilized by specialized macromolecules, and her group has used this for bottom-up fabrication of micropatterned single crystals.

Next, Aizenberg described the microlens arrays found in brittle stars that change color based on whether it is day or night. This was used as the inspiration to create synthetic tunable microlens arrays with pores by combining polymer synthesis, optics, and microfluidics. She discussed lessons learned in actuation at the nanoscale. The surfaces of Echinoderms are covered with moving spines and pedicellaria, which are environmentally responsive microactuators that provide a very effective antifouling mechanism. Aizenberg and her co-workers were able to replicate this using arrays of isolated, high-aspectratio rigid structures along with hydrogels. In the contracted state of the hydrogel, the nanocolumn structures were bent, but in the swollen state, the nanocolumns stood straight. This was used to create superhydrophobic-hydrophilic transition structures as well as responsive "nanograss."

Aizenberg also described the hierarchical self-assembly of soft, high-aspect ratio pillars standing on a wafer. Based on the level of the bending force, capillary force, and pillar length, different regimes can be described whereby neighboring pillars join and twist around each other. These groups of pillars then also join up and twist around each other and form larger assemblies, leading to a hierarchy of chiral twisting. The handedness of the chirality could also be controlled. This was used for particle trapping of polystyrene spheres. Aizenberg concluded that these bioinspired architectures have enormous potential for a variety of future applications.

\section{Materials Research Intersects Archaeology and Astronomy to Solve Mysteries}

During the lunch time series in Symposium $\mathrm{X}$ on Frontiers of Materials Research, two of the speakers presented

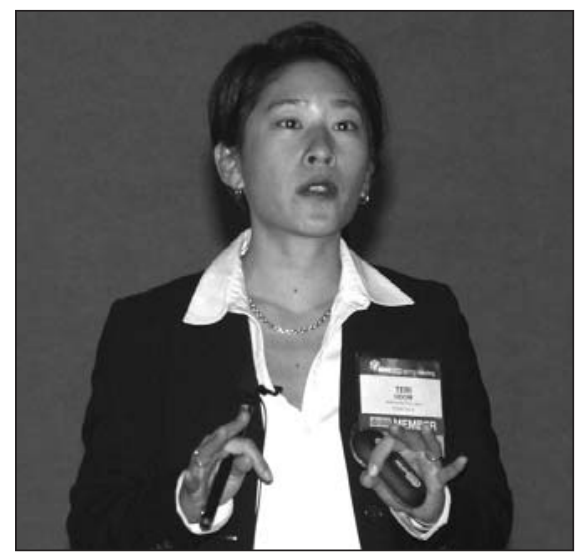

MRS 2009 Outstanding Young

Investigator Teri W. Odom

(Northwestern) discusses quasi-3D

plasmonic lattices.

overviews on how materials research has been used to help solve mysteries. One mystery involves the formation of ancient Egyptian pigments, and the other, the formation of comet 81P/Wild 2.

The ancient Egyptians mastered the art and science of the preparation of synthetic blue and green pigments as well as making a number of advances in the utilization of naturally occurring mineral pigments. The combination of these approaches to color resulted in the ancient Egyptians having the most diverse pigment palette of any ancient civilization. In a discussion of the color blue, for example, David Scott of the Cotsen Institute of Archaeology, University of California at Los Angeles, explained that Lapis Lazuli is the mineral typically used but it is rare. Also Azurite, which is a copper carbonate, is difficult to obtain. The Egyptians got around this by creating Egyptian blue, which was the world's first known synthetic pigment, Scott said. However, knowledge of Egyptian blue was lost around AD 6-7th century. Through collaborative work with the Metropolitan Museum of Art, the Petrie Museum, University College-London, and the Museum of Man in San Diego, scientists have now determined that Egyptian blue consists of calcium copper silicate, a phyllosilicate with sheets of six-membered rings with a tetragonal structure.

To solve a mystery of a different nature, in January of 2004, the Stardust NASA mission intercepted Comet $81 \mathrm{P} /$ Wild 2 and collected particles from the tail of the comet. Hundreds of micron-sized particles were returned to Earth early in 2006 and have been characterized by an international collaboration of physicists, chemists, and materials scientists. The goal of the mission, said Sean Brennan of Stanford University, was to study materials that had formed in a region of the solar system too far from the Sun to have been affected by its radiation. Scientists expected Comet $81 \mathrm{P} /$ Wild 2 to be a pristine remnant of the nebular material that formed the solar system because it has spent most of the last 4.6 Gyr in the Kuiper Belt beyond the orbit of Neptune, about 50 astronomical units (AU) from the Sun. The Stardust mission, launched in 1999, met the comet in the region between Mars and Earth and collected particles streaming from the comet (due to the heat of the sun) during a $3 \mathrm{~min}$. time period. The cometary material, consisting of grains ranging from $10 \mu \mathrm{m}$ down to $<2 \mathrm{~nm}$, was collected in aerogel, a very low density $\left(\sim 3 \mathrm{mg} / \mathrm{cm}^{3}\right)$ silica foam, to minimize the effects of deceleration from $6.1 \mathrm{~km} / \mathrm{s}$. The comet samples have been studied by a wide variety of analytical instruments including transmission electron microscopy, secondary ion mass spectrometry, and x-ray diffraction. The most surprising results of these studies, said Brennan, are the similarities and differences between Comet 81P/Wild 2 and meteorites collected in low-earth orbit. The cometary grains contain mineral crystal structures that require high temperatures to be formed (in some cases, >1400 K), completely inconsistent with their presumed formation 50 AU from the Sun. Yet the heterogeneity of the minerals found in the cometary grains is also inconsistent with any but the most primitive of meteorites. These results can only make sense if the materials found in Comet 81P/Wild 2 were formed close to the Sun after it ignited and then transported out to their location in the Kuiper Belt, Brennan said. He said that this result is the first solid evidence for "radial transport" during solar system formation, as postulated by several theoretical astrophysicists.

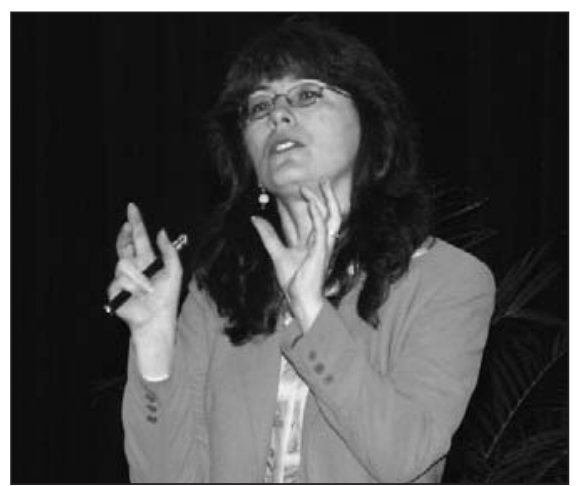

Joanna Aizenberg (Harvard) presents the 2009 Kavli Distinguished Lecture in Nanoscience. 


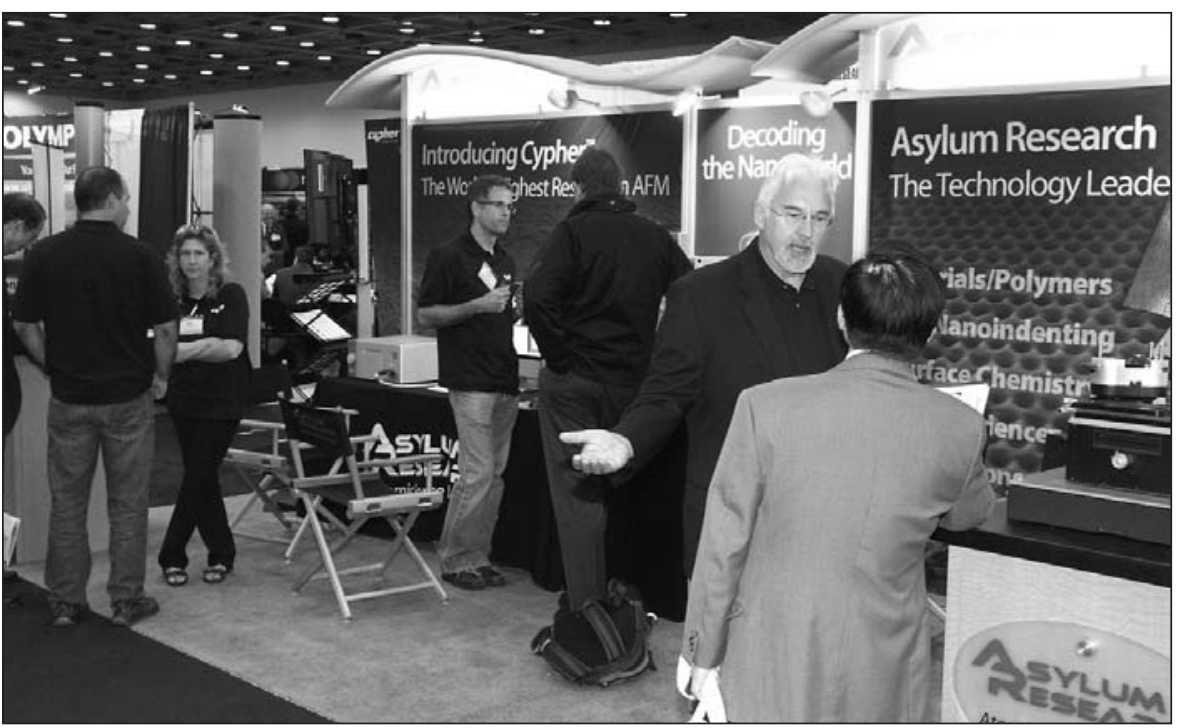

MRS Meeting attendees meet with equipment exhibitors to explore new products.

\section{Women in MS\&E Breakfast Focuses on Career Advancement}

The Women in Materials Science and Engineering Breakfast featured speaker Gregg A. Zank, Vice President, Chief Technology Officer, and Executive Director of Science and Technology at Dow Corning Corporation. Basing his presentation on Dow Corning's success, Zank talked about the many ways materials enable various industries, including the electronics industry and the photovoltaic industry. Both provide "huge materials challenges with exciting opportunities at the same time," he said. These challenges led to career paths in materials research that require collaborative efforts and the ability to bridge across multiple disciplines. Zank ended his presentation with some advice on career advancement: Find what you like doing, then figure out how to get paid for it, he said. Balance management and technology as well as balance work and home-life. While some scientists may be tempted to focus on what they can "do better" than their boss, Zank said he has focused on and learned from what his boss could do better. And timing is everything, he said: Typically, when companies offer an employee a new opportunity, they are investing in the employee for a broader goal. He has heard one CEO say that, during the course of his career, the only opportunity he turned down was one he has already done.

The Women in MS\&E Breakfast is organized by the MRS Outreach Committee and sponsored by Aldrich Materials Science.

\section{U.S. Government Funding Seminars Reveal Renewed Opportunities}

At each MRS Meeting, several U.S. government seminars are offered, providing updates on initiatives and budget changes that support basic research in materials. This year sees renewed effort in materials research and development (R\&D), from curiosity- to mission-driven, and agencies are eager to move the funding out to the universities and national laboratories.

From the Division of Materials Research (DMR) within the National Science Foundation, Zakya H. Kafafi's maxim is that "we cannot afford to 'move' [forward], we need to race." With an additional \$3 billion going to NSF from the recently passed American Recovery and Reinvestment Act (ARRA), on top of the FY2009 appropriations of $\$ 6.85$ billion, NSF now has a substantial amount of funding to race out the door in FY2009. Kafafi, who directs DMR, said emphasis is

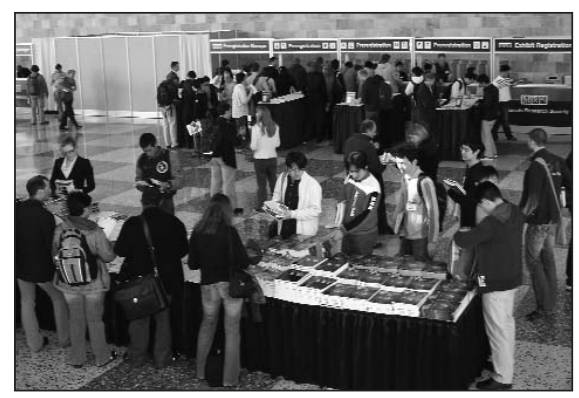

2009 Materials Research Society Spring Meeting. being given to fund investigators early in their careers, with a focus on broadening participation. Programs such as Partnerships for Research and Education in Materials (PREM) will expand to institutions primarily serving women and individuals with disabilities. DMR will expand its international activities. The next "MRSECs" (Materials Research Science and Engineering Centers) will expand activities to the international arena and the cyber infrastructure will be developed between research and education centers. DMR is continuing its Materials World Network and its International Materials Institutes. Among the many research topics supported by DMR, Kafafi described the SOLAR initiative, the Biomaterials Program, and plans to support siteindependent, technology-neutral R\&D for Next-Generation Coherent Light Sources.

The Department of Energy (DOE) Office of Basic Energy Sciences (BES) in the Office of Science will use its share of the increased funding from the ARRA and FY2009 budget to further its stated priorities, which include investing in science to achieve transformational discoveries, changing the landscape of energy demand and supply, and promoting economic prosperity through the creation of millions of "green" jobs. Tof Carim, program manager of the Scientific User Facilities Division in DOE, presented the overall DOE seminar as well as an additional session focused on the DOE Nanoscale Science Research Centers (NSRCs). BES maintains two interrelated research portfolios, investing in large, interdisciplinary research through the DOE national laboratories, and emphasizing innovationdriven science through its university grant program. The NSRCs are user facilities promoting interdisciplinary research among scientists studying nanoscale phenomena. The five user facilities are designed to provide specialized equipment and support staff at no cost to researchers through a peer-reviewed proposal process.

The goal of seeking "unprecedented materials properties" reverberated across the Department of Defense (DoD) government seminars this year. David Stepp of the Materials Science Division of the Army Research Office (ARO) said the realization of unprecedented materials properties will enable revolutionary devices and systems for the soldier of 25 years from now. Among other research emphases, the Division is currently seeking to establish a new thrust in the area of directed selfassembly of reconfigurable materials.

Mihal Gross, a program officer in the Naval Materials Division of the Office of 
Naval Research, surveyed the current technical areas of interests in the division, noting an overriding goal of fostering world-class research with game-changing potential for the Navy. The Division's programs cover a full spectrum from longrange, fundamental science and engineering research in the design and realization of new materials and systems with unprecedented properties and performance to facilitating technology transitions of research to fulfill the unique requirements of the Navy, Marine Corps, and Defense Department.

In the Air Force Office of Scientific Research (AFOSR) seminar, Michelle E. Ewy, a program manager of Aerospace, Chemical and Materials Sciences, said that AFOSR focuses on identifying breakthrough research opportunities in the
United States and abroad, fostering revolutionary basic research for air force needs, and transitioning technologies to DoD and industry.

From the National Institute of Standards and Technology, Michael Schen, senior scientific advisor to the director of the Technology Innovation Program (TIP), described the mission, objectives, and activities of the new TIP program. After the presentation, he and David Swanson, General Business Specialist in the program, fielded questions from the audience. Schen said that TIP promotes technological innovation by providing funding support to challenging, high-risk research projects that address critical national needs. The merit-based, competitive program can fund R\&D projects by single small-sized or medium-sized businesses or by joint ventures that also may include institutions of higher education, nonprofit research organizations, and national laboratories. The 2009 TIP competition is open to projects developing new technologies for the practical application of advanced materials, including nanomaterials, advanced alloys, and composites, in manufacturing; and the monitoring or retrofit of major public infrastructure systems, including water systems, dams and levees, and bridges, roads, and highways.

For further details on the research results reported at the 2009 MRS Spring Meeting, including symposium summaries, see the expanded Meeting coverage at www. mrs.org/S09MeetingReport. Proceedings as well as additional meeting highlights are available at Web site www.mrs. org/S09.

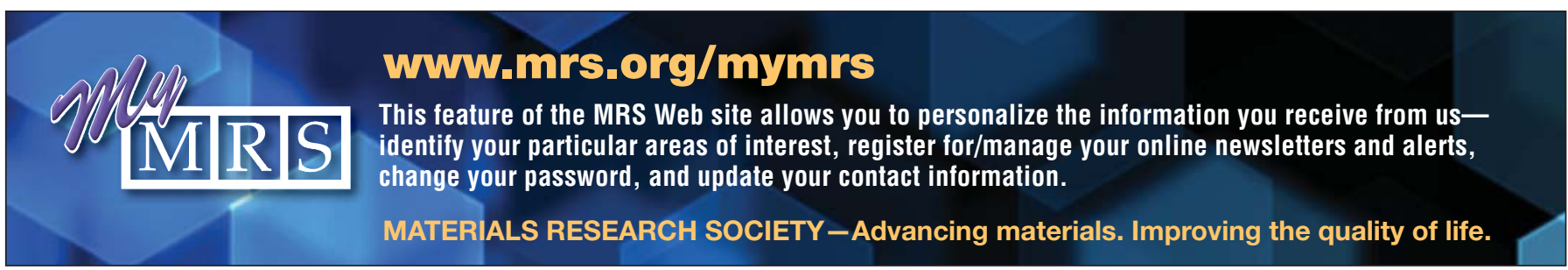

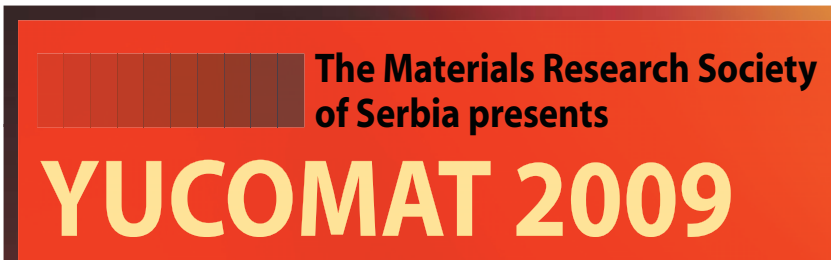

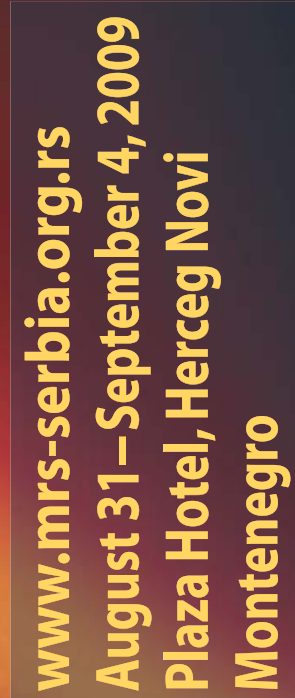

\section{Technical symposia include:}

- Advanced Methods in Synthesis and Processing of Materials

- Advanced Materials for High-Technology Application

- Nanostructured Materials

- Composites

- Biomaterials

- Plus three Plenary Sessions with 21 lectures, an Equipment Exhibit, and Awards.

More information can be obtained from the Meeting Web site or from Conference Secretary Aleksandra Stojicic at e-mail its@itn.sanu.ac.rs.

\section{$\mathrm{JAN}$ IS}

\section{Cryogen Free Probe Stations}

- $<5 \mathrm{~K}-650 \mathrm{~K}$ cryocooler based systems, using no liquid cryogens

- Fully vibration isolated sample mounting stage for sub-micron stability

Two to six probes, DC to $67 \mathrm{GHz}$ frequency range, with fiber optic option

Zoom optics with camera, monitor and full system integration

- Complete packages including probe station, temperature controller, optics, and vacuum pump

- LHe and $\mathrm{LN}_{2}$ cooled, and room temperature probe stations also available

\section{Janis Research Company}

2 Jewel Drive Wilmington, MA 01887 USA TEL +1 978 657-8750 FAX +1978 658-0349 sales@janis.com Visit our website at WWW.janis.com 